\title{
Phenotypic and genotypic detection of carbapenemase production among gram negative bacteria isolated from hospital acquired infections
}

Sreeja K. Vamsi, MSc (Medical), Rama S. Moorthy, PhD, Mary N. Hemiliamma, MD, Rama B. Chandra Reddy, PhD, Deepak J. chanderakant, PhD, Shravani Sirikonda, MBBS.

\begin{abstract}

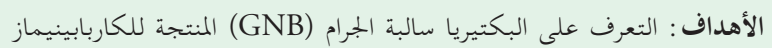

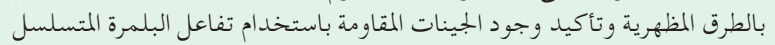
في الوقت الحقيقي (PCR)

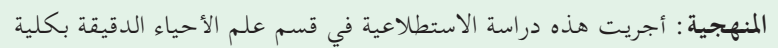

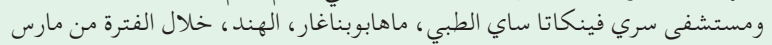

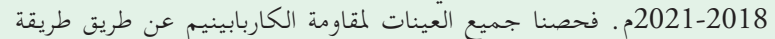

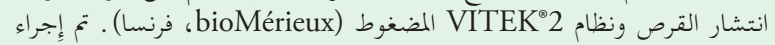



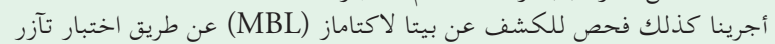

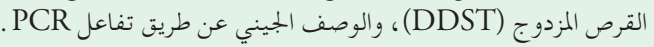

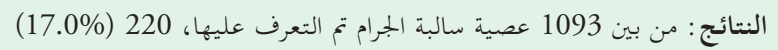

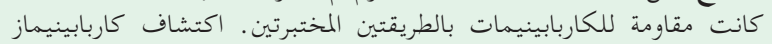

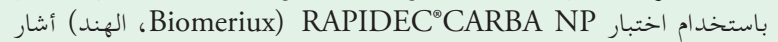
إِلى أن 207 (94.0\%) من منتجي كاربابينيماز ، منهم 189 (91.2\%) من (91.

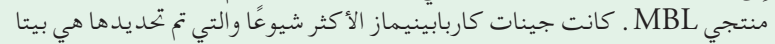

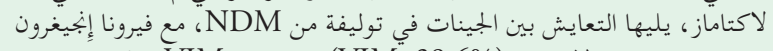

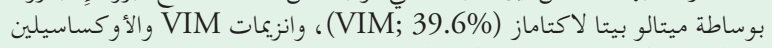

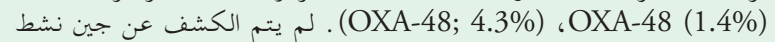



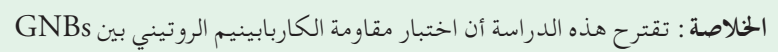

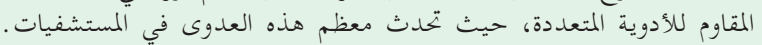

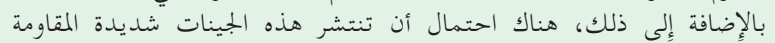

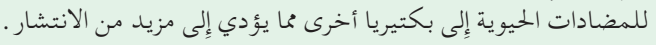

Objectives: To identify the carbapenemase producing Gram-negative bacteria (GNB) by phenotypic methods and to confirm the presence of resistant genes using realtime polymerase chain reaction (PCR).

Methods: This was a prospective study carried out at the Department of Microbiology, Sri Venkata Sai Medical College and Hospital, Mahabubnagar, India, from March 2018-2021. All samples were screened for carbapenem resistance by disc diffusion method and the VITEK $^{\circ} 2$ compact system (bioMérieux, France). Detection of carbapenemase was carried out using RAPIDEC ${ }^{\circ}$ CARBA NP test (Biomeriux Private Limited, South Delhi, India), screening for metallo- $\beta$-lactamases (MBL) was carried out by double disk synergy test (DDST), and genotypic characterization by real-time PCR.

Results: Among the 1093 Gram-negative bacilli identified, $220(17.0 \%)$ were resistant to carbapenems by both tested methods. Carbapenemase detection using the RAPIDEC ${ }^{\circ}$ CARBA NP test indicated that 207 (94.0\%) were carbapenemase producers, of which 189 (91.2\%) were MBL producers. The most common carbapenemase genes identified were New Delhi metallo- $\beta$-lactamase (NDM; 47.3\%), followed by the co-existence of genes in combination of NDM, with Verona integron-mediated metallo- $\beta$-lactamase (VIM; 39.6\%), VIM and oxacillin hydrolyzing enzymes-48 (OXA-48; 4.3\%), and OXA$48(1.4 \%)$. No gene of active on imipenem, Klebsiella pneumonia carbapenemase, VIM, or OXA-48 alone was detected.

Conclusion: This study suggests routine carbapenem resistance testing among multi-drug resistant-GNBs, as most of these infections occur in hospitals. In addition, there is a possibility that these highly antibiotic-resistant genes could spread to other bacteria resulting in further dissemination.

Keywords: antibacterial agents, carbapenems, betalactamases, drug resistance, microbial

Saudi Med J 2022; Vol. 43 (3): 236-243 doi: 10.15537/smj.2022.43.3.20210809

From the Department of Microbiology (Sreeja Vamsi); Manipal Academy of Higher Education; Karnataka, from the Department of Microbiology (Moorthy); Palamur Biosciences Pvt. Ltd., from the Department of Microbiology (Hemiliamma, Chandra Reddy); from the Department of Community Medicine (chanderakant); and from the Department of Microiology (Sirikonda); Sri Venkata Sai Medical College and Hospital, Telangana, India.

Received 8th October 2021. Accepted 15th February 2022.

Address correspondence and reprint request to: Dr. Rama S. Moorthy, CEO, Department of Microbiology, Palamur Biosciences Pvt. Ltd., Telangana, India.E-mail:mail-ram.murthy@palamurbio.com ORCID ID: https://orcid.org/0000-0001-9704-5886 
$\mathrm{H}$ ospital-acquired infections (HAI) are defined as infections that occur after 48 hours or more of hospital entry. Hospital infections include; central lineassociated bloodstream infections, ventilator-associated pneumonia, catheter-associated urinary tract infection, and surgical site infection. The major contributors to hospital-acquired infections include several factors such as, prolonged hospital stay, invasive measures, ventilator support, immune suppression, old age, diabetes, and stay in the intensive care unit, indwelling devices, and recurrent visits to hospitals. Morbidity and mortality are the most important challenges associated with HAI. ${ }^{1,2}$

Carbapenem drugs are the most valuable drugs for treating multi-drug resistant Gram-negative bacteria (MDR-GNB) infections and have been used for the past 10 years. However, there has been a significant growth of carbapenem-resistant organisms that cause severe damage to public health. ${ }^{3,4}$ These carbapenems, which include meropenem, ertapenem, and imipenem, are $\beta$-lactam antibiotics that possess a $\beta$-lactam ring and have a wide range of activity and great usefulness. These antibiotics are used as a last line of defense for treating infections caused by MDR-GNBs as well as organisms that produce extended-spectrum $\beta$-lactamases, which are used to screen carbapenem resistance in laboratories, and develop resistance either through gene transfer or mutations. ${ }^{5,6}$

Carbapenem resistance is mainly owing to the expression of a carbapenemase enzyme, efflux pump, or porin loss. Among these, the most important and difficult mechanism is the production of the carbapenemase enzyme, because it is present on mobile genetic elements, which are easily transferable from one bacterium to another bacterium such as Pseudomonas spp., Acinetobacter spp., Escherichia coli (E. coli), and Klebsiella spp., which the World Health Organization (WHO) has designated as high priority organisms in 2017..$^{7-10}$ The major carbapenemase genes are bla-Klebsiella pneumonia carbapenemase (blaKPC), bla-oxacillin hydrolyzing enzymes-48 (blaOXA-48), bla-New Delhi metallo- $\beta$-lactamase (blaNDM), bla-Verona integron-mediated metallo- $\beta$-lactamase (blaVIM), and bla-active on imipenem (blaIMP), which are present globally and cause nosocomial infections. Many researchers have studied various

Disclosure.This study was funded by the Department of Science and Technology, Government of India, New Delhi, India, as part of the Women Scientist Scheme (no.: SR/WOS-A/LS-643/2016 [G]). methods of carbapenem resistance detection including carbapenemases. ${ }^{8,11,12}$ Methodologies for the detection of carbapnem resistance range from conventional disc diffusion and minimum inhibitory concentration (MIC) determination methodologies to advanced and rapid detection methods such as automated methods, RAPIDEC ${ }^{\circ}$ CARBA NP (Biomeriux Private Limited, South Delhi, India), and genotypic methods using polymerase chain reaction (PCR). Many of these advanced methodologies are not routinely used in peripheral and resource poor settings because of which detection of these resistant organisms is missed or delayed which in turn, leads to therapeutic failure and dissemination of these resistant strains in the hospitals. Identifying the prevalence of these carbapenemresistant Gram-negative bacilli (CRGNB) in any hospital will help to formulate the appropriate infection control guidelines which prevent further spread of these infections and also economical burden on the hospitals.

As a result, we used genotypic approaches to estimate the prevalence of $\beta$-lactamase Gram-negative microorganisms CRGNB and to identify carbapenemproducing isolates, which are important in choosing empirical therapy, designing good antibiotic policies, updating local antibiotic guidelines for doctors, and in determining clinical treatment failure. With this background, this study aimed to screen for carbapenemase production among HAI using both phenotypic and genotypic methods.

Methods. This was a prospective study carried out in the Department of Microbiology, Sri Venkata Sai (SVS) Medical College and Hospital, Mahabubnagar, India, over a period of 3 years from March 2018-2021. A certification of Ethical Clearance (SVS Medical College/Institutional Ethical Committee; approval no.: 05/2018-623) was obtained and the study was carried out in accordance to the principles of Helsinki Declaration.

All the inpatient's samples with suspected HAI showing growth of laboratory confirmed CRGN bacteria such as E. coli, Klebsiella spp., Acinetobacter spp., Pseudomonas spp., and Enterobacter spp. isolates were included in the study. Samples with carbapenem sensitive organisms, clinically insignificant growth characters, and other organisms other then the mentioned were excluded from the study.

A total of 1093 Gram-negative bacteria that were isolated from various clinical samples, such as urine, pus, stool, sputum, blood, endotracheal secretions, cerebrospinal fluid, and other body fluids from hospitalized patients were processed at the Department 
of Microbiology, SVS Medical College and Hospital and were identified to the species level based on the Gram staining, colony morphology, and biochemical reaction. These isolates were screened for carbapenem resistance using the Kirby Bauer disc diffusion method.

The results were interpreted according to the Clinical Laboratory Standards Institute (CLSI-M1OO-S29) guidelines. ${ }^{13}$ Organisms that were resistant to imipenem and meropenem were further processed to determine their MICs using the $\mathrm{VITEK}^{\oplus} 2$ compact system (bioMérieux, France). ${ }^{14}$ Antibiotic resistance was detected for antibiotics such as cefoxitin, cefepime, ceftazidime, ceftriaxone, piperacillin, tazobactam, imipenem, meropenem, ciprofloxacin, ertapenem, gentamicin, amikacin tigecycline, colistin, cefoperazone sulbactum, and cotrimoxazole (Himedia, Mumbai, India). Klebsiella pneumonia ATCC BAA 1705 and ATCC BAA 1706, Escherichia coli ATCC 25922 were used as the positive and negative controls for phenotypic and genotypic investigations.

Detection of carbapenems production. Imipenem and meropenem resistant organisms were used in further investigations.

RAPIDEC ${ }^{\oplus}$ CARBA NP test. The test was carried out as described by Nordmann, Poirel, and Dordet "A change in color of the $\mathrm{Ph}$ indicator is indicative of carbapenem hydrolysis caused by carbapenemaseproducing bacteria, which produce acid". ${ }^{15}$ The procedure followed the manufacturer's instructions. API suspension medium $(25 \mu \mathrm{L}$; bioMérieux, New Delhi, India) was added to the wells and 5-6 colonies from the fresh culture plate were obtained and added to the prescribed well. The turbidity of the inoculum was compared with the provided strip. Next, $10 \mu \mathrm{L}$ of the inoculum was added to 2 wells of which one well contained imipenem. Imipenem was used as the carbapenemase zinc substrate for metallo- $\beta$-lactamases (MBL)-producing gram-negative bacteria and the results were considered positive if the color changes from red to yellow, orange, or thick orange in comparison to the control well. Organisms that tested positive for carbapenemase were used for further evaluations. ${ }^{15}$

Double disk synergy test. Phenotypic testing was carried out following the guidelines of the CLSI. Briefly, 0.5 Mac Farland bacterial inoculum was spread on a Muller Hinton Agar plate as a lawn culture and one imipenem disk was placed at the center with a blank disc place adjust $20 \mathrm{~mm}$ apart. Next, $10 \mu \mathrm{L}$ of 0.5 ethylene-diamine-tetraacetic acid $(750 \mu \mathrm{g})$ was inoculated on the blank disc and incubated at $37^{\circ} \mathrm{C}$ for 24 hours. Presence of an inhibitory zone was considered MBL-positive, and cultures that did not exhibit such zones were considered as the Serine group. ${ }^{16}$
Genotyping method. The overnight bacterial culture was used for DNA extraction using the Hi Per Bacterial Genomic DNA Extraction Kit (Hi-Media HTBM009) in accordance with the manufacturer's instructions.

Bacteria DNA was isolated and eluted from the columns in $200 \mu \mathrm{L}$ elution buffer and stored in a mini Eppendorf tube at $-20^{\circ} \mathrm{C}$ until further use.

The carbapenemase gene (multiplex) probe-based Hi-Media Hi-PCR kit was used to detect specific regions of the gene encoding the carbapenemase enzyme. The technique is very easy, fast, and powerful for the detection of carbapenemase genes such as blaVIM, blaNDM, blaKPC, blaOXA-48, and blaIMP and can be used to accurately identify one or a combination of carbapenemase genes in a single tube reaction with a large variety of organisms. Positive, internal, and negative controls were used as specified in the manufacturer's instructions. Briefly, the multiplex probe PCR kit is designed to detect specific regions of the genes encoding various carbapenemase enzymes. There are 2 master mixes in this kit, wherein master mix-1 detects NDM, KPC, IMP, and VIM in the FAM, HEX, Texas Red, and Cy5 channels, where as master mix-2 detects OXA-51, OXA-23, OXA-48, and OXA-58 in the FAM, HEX, Texas Red, and Cy5 channels. The internal control was detected in the Cy5.5 channel in both the master mixes. The kit allows sensitive and specific detection of single and co-present carbapenemase-encoding genes in a single tube reaction. The cycling method consisted of initial denaturation at $95^{\circ} \mathrm{C}$ for 10 minutes, followed by denaturation at $95^{\circ} \mathrm{C}$ for 5 seconds, and 45 cycles of annealing and extension at $60^{\circ} \mathrm{C}$ for one minute, and the final holding stage was carried out in Quant Studio real-time PCR (ThermoScientific). A cycle threshold value of $\leq 40$ and band was considered positive (Figure 1).

Statistical analysis. Data obtained were analyzed using descriptive statistics, Chi-square test carried out using Statistical Packaage for the Social Sciences, version 23.0 (IBM Corp., Armonk, NY, USA). A $p$-value of $<0.05$ was considered significant.

Results. Among the 1093 Gram-negative bacilli identified, $220(17 \%)$ by using the disc diffusion method and VITEK 2 methods, they were shown to be resistant to carbapenems. The carbapenemase enzyme was detected in these species utilizing the RAPIDEC ${ }^{\circ}$ CARBA NP test, in which 207 (94.0\%) were identified as carbapenemase producers. Among these, 189 (91.2\%) were MBL producers and 18 (8.6\%) were non-MBL producers by the double disk synergy test (DDST) method. 
Among all the carbapenemase producers, the predominant organisms were Klebsiella spp. (56.7\%), followed by E. coli (17.7\%), Acinetobacter spp. (11.4\%), Pseudomonas spp. (10.9\%), and Enterobacter (3.1\%).

In the 207 carbapenemase-producing isolates, carbapenemase genes were detected in 192 (92.7\%) isolates using real-time PCR. The most common genes identified were NDM (47.3\%), followed by the co-existence of genes in combination of NDM, with VIM (39.6\%), VIM and OXA-48 (4.3\%), and OXA-48 (1.5\%). No gene of IMP, KPC, VIM, or OXA- 48 alone was detected.

Among the NDM gene-positive organisms, Klebsiella (58.2\%) was the most common organism, followed by E. coli (22.4\%), Acinetobacter (9.2\%), Pseudomonas (8.2\%), and Enterobacter (2.0\%). The coexistence of VIM and NDM appeared predominantly in Klebsiella (57.3\%), followed by E. coli $(14.6 \%)$, Acinetobacter (12.2\%), Pseudomonas (11.0\%), and Enterobacter (4.9\%). Klebsiella and Pseudomonas exhibited the co-existence of OXA-48 and NDM, and 3 genes (NDM, VIM, and OXA-48) were co-expressed in Klebsiella (44.4\%), Acinetobacter (33.3\%), and Pseudomonas (22.2\%) isolates (Table 1).

The majority of the organisms were isolated from endotracheal secretions (23.9\%), followed by pus and wound swabs $(21.3 \%)$, blood $(22.3 \%)$, urine $(8.8 \%)$, sputum $(13.0 \%)$, stool $(7.8 \%)$, cerebrospinal fluid (1.5\%), and other body fluids (1.0\%). The most predominant organisms in endotracheal secretions were Klebsiella (58.7\%) and Acinetobacter (15.2\%). The predominant organisms in the pus samples were Klebsiella (65.9\%), followed by Pseudomonas (17.1\%). In urine samples, $E$. coli was predominant $(41.2 \%)$, whereas, in sputum samples it was Klebsiella $(80.0 \%)$ and in stool samples, it was E. coli $(80.0 \%)$. Based on statistical analysis, it was found that the prevalence of carbapenems-resistant isolates differed significantly across other type of samples ( $p<0.05$; Table 2$)$.

The prevalence of carbapenemase genes was higher in the $0-21$ years age group with $88(45.8 \%)$ cases followed by the $21-40$ years with $52(27 \%)$ cases.

There was $100 \%$ resistance to cefoxitin, cefepime, ceftazidime, ceftriaxone, piperacillin-tazobactam, imipenem, and meropenem. Resistance to further antibiotics was varied, ciprofloxacin $(96.7 \%)$, ertapenem (91.0\%), gentamicin (89.0\%), amikacin (69.6\%), cefoperazonesulbactam (82.0\%), trimethoprim/ sulfamethoxazole $(64.0 \%)$, tigecycline $(6.14 \%)$, and colistin (4.7\%). A important variance was detected in the resistance of CRGN bacteria to numerous antimicrobial agents $(p<0.05$; Figure 2).

The pre-dominant genes in the blood isolates were NDM (55.8\%), NDM and VIM (41.9\%), followed by NDM, VIM, and OXA-48 (2.3\%). The dominant gene in cerebrospinal fluid samples was NDM and VIM
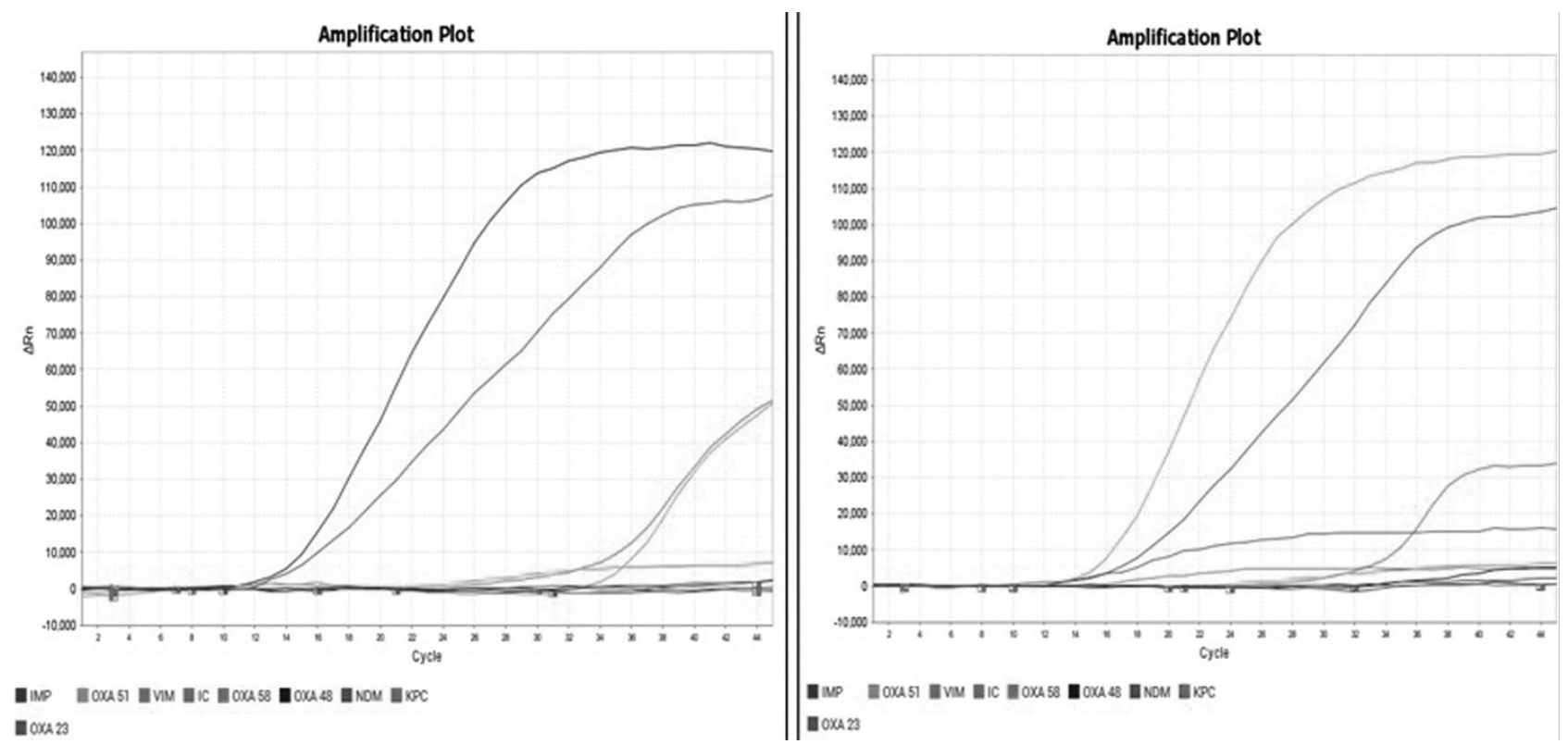

Figure 1 - Representing amplification plot of New Delhi metallo- $\beta$-lactamase (NDM), Verona integron-mediated metallo- $\beta$-lactamase (VIM), and oxacillin hydrolyzing enzymes-48 (OXA-48) by using Hi-media HiPCR carbapenemase gene (multiplex) probe PCR kit; identification of genes using real-time polymerase chain reaction. 
Table 1 - Prevalence of genes in different carbapenem-resistant isolates.

\begin{tabular}{lcccc}
\hline Organisms & NDM & NDM, VIM & $\begin{array}{c}\text { Genes } \\
\text { NDM, VIM, OXA-48 }\end{array}$ & NDM, OXA-48 \\
\hline Escherichia & $22(22.4)$ & $12(14.6)$ & - & - \\
Klebsiella & $57(58.2)$ & $47(57.3)$ & $4(44.4)$ & $2(22.2)$ \\
Pseudomonas & $8(8.2)$ & $9(11.0)$ & - & $2(66.3)$ \\
Enterobacter & $2(2.0)$ & $4(4.9)$ & $3(33.3)$ & - \\
Acinetobacter & $9(9.2)$ & $10(12.2)$ & $9(100)$ & $3(100)$ \\
Total & $98(100)$ & $82(100)$ & & - \\
\hline
\end{tabular}

NDM: New Delhi metallo-beta-lactamase, VIM: Verona integron-mediated metallo- $\beta$-lactamase, OXA-48: oxacillin hydrolyzing enzymes-48, n: number

Table 2 - Distribution of carbapenemase gene-producing organisms in different samples.

\begin{tabular}{|c|c|c|c|c|c|c|c|c|}
\hline \multirow[t]{2}{*}{ Organisms } & \multicolumn{8}{|c|}{ Samples } \\
\hline & Blood & CSF & ET & $\begin{array}{r}\text { Pus and wound swabs } \\
\mathrm{n}(\%)\end{array}$ & Urine & Sputum & Stool & Others \\
\hline Klebsiella & $23(53.5)$ & $1(33.3)$ & $27(58.7)$ & $27(65.9)$ & $6(35.3)$ & $20(80.0)$ & $3(20.0)$ & $2(100)$ \\
\hline Escherichia coli & $7(16.3)$ & - & $3(6.5)$ & $2(4.9)$ & $7(41.2)$ & $3(12.0)$ & $12(80.0)$ & - \\
\hline Enterobacter & $2(4.7)$ & - & $3(6.5)$ & $1(2.4)$ & - & - & - & - \\
\hline Acinetobacter & $9(20.9)$ & - & $7(15.2)$ & $4(9.8)$ & $1(5.9)$ & $1(4.0)$ & - & - \\
\hline Pseudomonas & $2(4.7)$ & $2(66.7)$ & $6(13.0)$ & $7(17.1)$ & $3(17.6)$ & $1(4.0)$ & - & - \\
\hline Total & $43(100)$ & $3(100)$ & $46(100)$ & $41(100)$ & $17(100)$ & $25(100)$ & $15(100)$ & $2(100)$ \\
\hline
\end{tabular}

(66.7\%), followed by NDM (33.3\%). In endotracheal secretions, the co-existence of NDM and VIM was prevalent $(45.7 \%)$, followed by that of NDM (41.3\%). In pus and wound swabs, $19(46.3 \%)$ of the most prevalent genes were NDM and NDM with VIM. In sputum, the most common gene isolated was NDM (52.0\%), followed by NDM and VIM (44.0\%). In stool, the pre-dominant gene was NDM $(80.0 \%$; Table 3).

Discussion. Carbapenem is the medication of choice for the treatment of extended-spectrum $\beta$-lactamases and MDR organisms. Resistance to carbapenems is progressively being observed, especially in HAI, which are difficult to treat, pose a huge economic burden and are allied with improved mortality and morbidity. ${ }^{17}$

To decrease the threat of infection spreading in the hospital and to reduce mortality rates, this study reconnoitered the existence of carbapenemase-producing organisms using phenotypic methods and determined the distribution of carbapenemase genes by genotypic testing. The prevalence of carbapenem resistance was $17.0 \%$ in this study, which is similar to Haji et al ${ }^{18}$ 's study. The incidence of prevalence in various parts of India varies from $14-69 \%$. This was mostly owing to infection control practices, hospital infrastructure, and the number of antibiotics used. ${ }^{19}$

One of the major goals of this research was to see whether carbapenemase-genes were present in gramnegative bacteria isolated form hospitalized patients. Carbapenemase-producing micro-organisms are difficult to detect and require phenotypic and genotypic analyses. Of the 220 isolates, 207 (94.0\%) had phenotypic positivity, which was comparable to that reported by Diwakar et al. ${ }^{20}$ Our genotypically positive findings $(87.2 \%)$ were comparable to the genotypically positive rate of $90.3 \%$ reported by Garg et al. ${ }^{21}$ The major differences were likely due to geographic region, the testing method used, and the organism. ${ }^{22}$

New Delhi metallo- $\beta$-lactamase $(47.3 \%)$ was the pre-dominant gene in this study, which was similar to that reported by Naim et al. ${ }^{23}$ The prevalence of resistance genes was higher in Klebsiella spp. (56.7\%) followed by $E$. coli $(17.7 \%)$. The prevalence of NDM in India is causing major public health challenges due to its elevated medical and economic burden. ${ }^{24} \mathrm{We}$ found that blaKPC, blaSIM, and blaIMP genes were not detected, which is consistent with the observations made by Garg et al. ${ }^{21}$ 


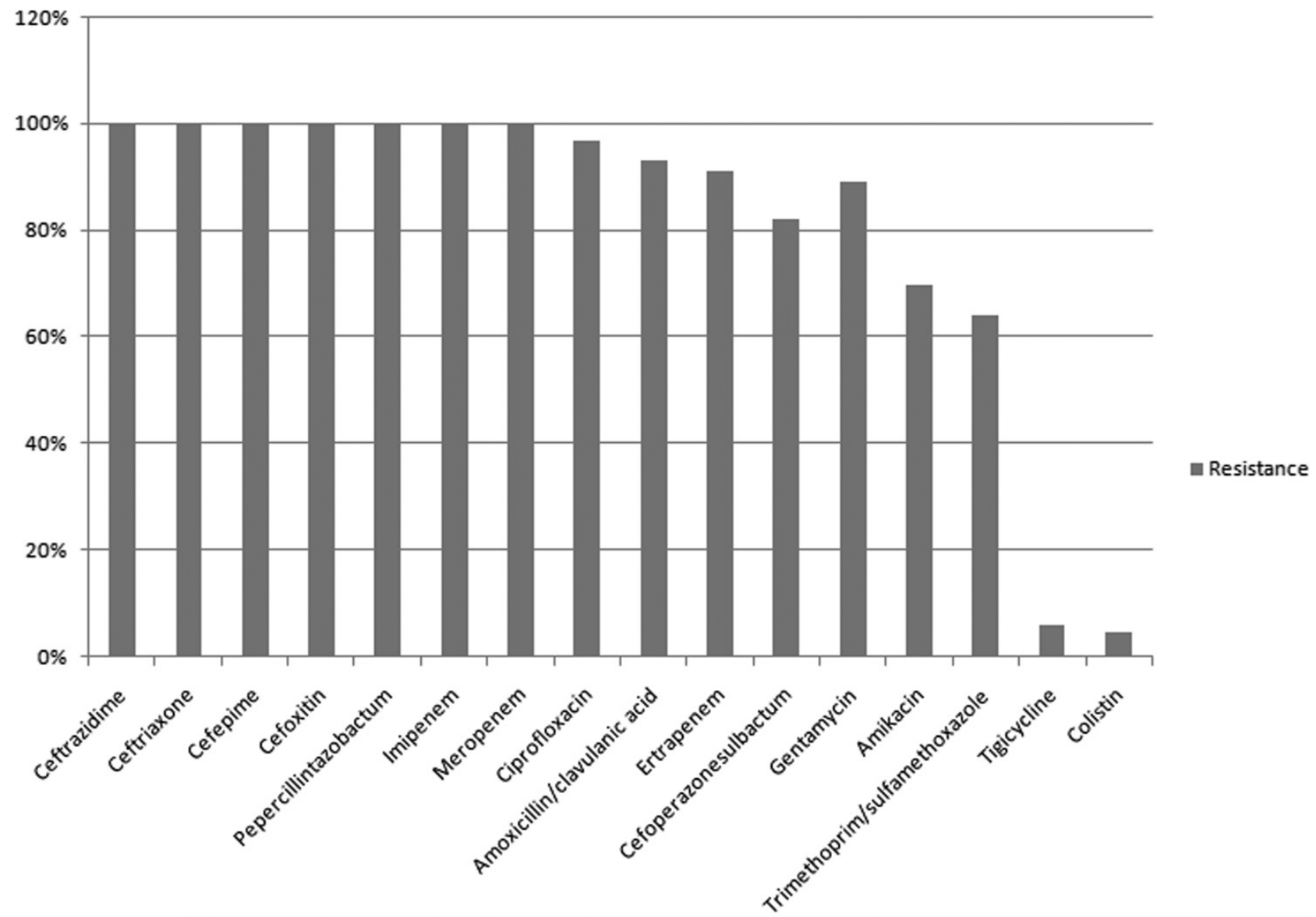

Figure 2 - Resistance pattern of isolates harboring carbapenemase genes. represents the resistance pattern to various drugs by carbapenemase producing Gram negative bacteria.

Table 3 - Distribution of carbapenemase genes in different samples.

\begin{tabular}{|c|c|c|c|c|c|c|c|c|}
\hline \multirow[t]{2}{*}{ Genes } & \multicolumn{8}{|c|}{ Samples } \\
\hline & Blood & CSF & ET & $\begin{array}{c}\text { Pus and wound swabs } \\
\text { n (\%) }\end{array}$ & Urine & Sputum & Stool & Others \\
\hline NDM & $24(55.8)$ & $1(33.3)$ & $19(41.3)$ & $19(46.3)$ & $9(52.9)$ & $13(52.0)$ & $12(80.0)$ & $1(50.0)$ \\
\hline Co-existence of NDM and VIM & $18(41.9)$ & $2(66.7)$ & $21(45.7)$ & $19(46.3)$ & $7(41.2)$ & $11(44.0)$ & $3(20.0)$ & $1(50.0)$ \\
\hline Co-existence of NDM, VIM, and OXA- 48 & $1(2.3)$ & - & $4(8.7)$ & $3(7.4)$ & - & $1(4.0)$ & - & - \\
\hline Co-existence of NDM and OXA- 48 & - & - & $2(4.3)$ & - & $1(5.9)$ & - & - & - \\
\hline Total & $43(100)$ & $3(100)$ & $46(100)$ & $41(100)$ & $17(100)$ & $25(100)$ & $15(100)$ & $2(100)$ \\
\hline
\end{tabular}

In this study, the co-existence of VIM and NDM was 39.6\%, whereas Mohanam et $\mathrm{al}^{25}$ reported $14.6 \%$ and Ellappan et $\mathrm{al}^{26}$ reported a $17.3 \%$ co-existence in Pseudomonas aeruginosa from Southern India.

The co-existence of NDM and OXA-48 in this study was $1.4 \%$, whereas in a study by Garg et $\mathrm{al}^{21}$ it was
20.0\%. Grag et $\mathrm{al}^{21}$ identified the NDM and OXA-48 co-existance pre-dominantly in E.coli, followed by Klebsiella, and Enterobacter. None of the nonfermenters in his study exhibited OXA-48 gene. Whereas, according to Vatansever et $\mathrm{al}^{27}$ the co-harboring of OXA-48 and NDM in colistin-resistant Pseudomonas aeruginosa 
was $88.8 \%$. Males were more likely to be resistant to carbapenem (66.8\%), similar to that reported by Esther et al. $^{28}$ New Delhi metallo- $\beta$-lactamase prevalence in stool samples was approximately $7.8 \%$, which was slightly higher than the $3.6 \%$ reported by Pan et $\mathrm{al}^{29}$ and lower than the $18.5 \%$ reported by Esther et al. ${ }^{28}$ Moreover, these results confirmed that stool samples were colonized with carbapenemase genes, thus emphasized the need to screen for NDM. ${ }^{28,29}$ A large proportion of carbapenem-resistant organism's exhibit resistance to commonly used drugs. Most CRGNBs are still sensitive to tigecycline and colistin, which are the last lines of defense against CRGNB. We found that $18(8.6 \%)$ of the samples we evaluated were phenotypically positive. However, no genes were detected using real-time PCR. There may be a combination of factors contributing to this effect, such as gene targets in this study, a mutation, or loss of porin. Thus, it is important to accurately identify the genes responsible for carbapenemase production for better treatment outcome and also further research needs to be carried out to identify all the mechanisms of resistance co-existing in the resistant isolates, which if identified correctly can help in better management of patients by choosing appropriate antibiotics either alone or in combination for the treatment of these drug resistance infections.

Study limitation. The study was a single center study carried out in a tertiary care center and only resistance to carbapenems by carbapenmase production was studied. Role of other co-existing mechanism such as porin loss or efflux pumps in the causation of carbapenem resistance has not been evaluated.

In conclusion, carbapenemase genes are spreading rapidly worldwide due to the increased prevalence of horizontal transfer. As there are no new drugs available, and prevalence incidences vary regionally, real-time PCR probe-based detection of these genes is beneficial for early detection, developing infection control protocols, and promoting appropriate antibiotic use. The presence of co-existing carbapenemase genes is concerning. In addition, there is a possibility that highly antibiotic-resistant genes could spread to other bacteria because of their high spreading ability and thereby increase the possibility of further dissemination. Routine carbapenem resistance testing is suggested among MDR-GNBs at least in HAI as the prevalence was high which was shown in this study.

Acknowledgment. The authors gratefully acknowledge Manipal Academy of Higher Education, Karnataka, Palamur Biosciences Pvt. Ltd., and SVS Medical College, Mahabubnagar, India, for thier cooperation. We also would like to thank Dr. Murali for providing his comments on the draft version of the manuscript and Dr.Sreekanth for helping in the manuscript writing. We would like to thank Editage for English language editing.

\section{References}

1. Haque M, Sartelli M, McKimm J, Abu Bakar M. Health careassociated infections - an overview. Infect Drug Resist 2018; 11 : 2321-2333.

2. He Q, Wang W, Zhu S, Wang M, Kang Y, Zhang R, et al. The epidemiology and clinical outcomes of ventilator-associated events among 20,769 mechanically ventilated patients at intensive care units: an observational study. Crit Care 2021; 25: 44.

3. Paveenkittiporn W, Lyman M, Biedron C, Chea N, Bunthi C, Kolwaite A, et al. Molecular epidemiology of carbapenemresistant Enterobacterales in Thailand, 2016-2018. Antimicrob Resist Infect Control 2021; 10: 88.

4. Al-Ouqaili MTS, Al-Taei SA, Al-Najjar A. Molecular detection of medically important carbapenemases genes expressed by Metallo- $\beta$-lactamase producer isolates of Pseudomonas aeruginosa and Klebsiella pneumonia. Asian Journal of Pharmaceutics 2018; 12: S991-S1001.

5. Eatemadi A, Al Risi E, Kasliwal AK, Al Záabi A, Moradzadegan $\mathrm{H}$, Aslani Z. A proposed evidence-based local guideline for definition of multidrug-resistant (MDR), extensively drugresistant (XDR) and pan drug-resistant (PDR) bacteria by the Microbiology Laboratory. IJCSRR 2021; 4: 146-153.

6. Rea-Neto A, Youssef NC, Tuche F, Brunkhorst F, Ranieri VM, Reinhart K, et al. Diagnosis of ventilator-associated pneumonia: a systematic review of the literature. Crit Care 2008; 12: R56.

7. Breijyeh Z, Jubeh B, Karaman R. Resistance of Gram-negative bacteria to current antibacterial agents and approaches to resolve it. Molecules 2020; 25: 1340.

8. Shugart A, Mahon G, Huang JY, Karlsson M, Valley A, Lasure $\mathrm{M}$, et al. Carbapenemase production among less-common Enterobacterales genera: 10 US sites, 2018. JAC Antimicrob Resist 2021; 3: dlab137.

9. Sękowska A, Bogiel T, Kaczmarek A. Evaluation of the usefulness of selected methods for the detection of carbapenemases in Klebsiella strains. J Med Microbiol 2020; 69: 792-796.

10. World Health Organization. WHO global priority list of antibiotic-resistant bacteria to guide research, discovery, and development of new antibiotics. [Updated 2017; 2021 Aug 16]. Available from: https://www.aidsdatahub.org/resource/ who-global-priority-list-antibiotic-resistant-bacteria

11. Bouganim R, Dykman L, Fakeh O, Motro Y, Oren R, Daniel C, et al. The clinical and molecular epidemiology of noncarbapenemase-producing carbapenem-resistant Enterobacteriaceae: A case-case-control matched analysis. Open Forum Infect Dis 2020; 7: ofaa299.

12. Gajdács M, Ábrók M, Lázár A, Jánvári L, Tóth Á, Terhes G, et al. Detection of VIM, NDM and OXA-48 producing carbapenem resistant Enterobacterales among clinical isolates in Southern Hungary. Acta Microbiol Immunol Hung 2020; 67: 209-215.

13. Clinical and Laboratory Standards Institute. Performance standards for antimicrobial susceptibility testing, 20th informational supplement, Doc. M100ED29-2019. [Updated 2019; 2019 Jan]. Available from: https://clsi.org/

14. Cury AP, Girardello R, Duarte AJDS, Rossi F. KPC-producing Enterobacterales with uncommon carbapenem susceptibility profile in Vitek 2 system. Int J Infect Dis 2020; 93: 118-120. 
15. Walthall K, Anderson K, Reese N, Lonsway D, Rasheed JK, Karlsson M. Evaluation of the RAPIDEC CARBA NP, conventional CarbaNP, and the modified carbapenem inactivation method (mCIM) tests for phenotypic detection of carbapenemase-producing organisms. AJCP 2018; 150: S123-S124.

16. Suryavanshi KT, Lahiri KK. Assessment of RapidecCarba NP test, as screening test to detect carbapenemase, specially metallo$\beta$-lactamase producing Gram negative bacteria at tertiary care centre. IJHSR 2018; 8: 56-61.

17. Al-Ouqaili MTS, Khalaf EA, Al-Kubaisy SH. DNA sequence analysis of BlaVEB gene encoding multi-drug resistant and extended-spectrum $\beta$-lactamases producer isolates of Enterobacteriaceae and Pseudomonas aeruginosa. The Open Microbiology Journal 2020; 14: 40-47.

18. Haji SH, Aka STH, Ali FA. Prevalence and characterisation of carbapenemase encoding genes in multidrug-resistant Gramnegative bacilli. PLoS One 2021; 16: e0259005.

19. Mohammed MU, Manisha DR, Nagamani K. Clinical, phenotypic and genotypic profile of carbapenem resistant Gram negative infections in intensive care units. Indian J Microbiol Res 2021; 8: 28-34.

20. Diwakar J, Verma RK, Singh DP, Singh A, Kumari S. Phenotypic detection of carbapenem resistance in Gram-negative bacilli from various clinical specimens of a tertiary care hospital in Western Uttar Pradesh. Int J Res Med Sci 2017; 5: 3511-3514.

21. Garg A, Garg J, Kumar S, Bhattacharya A, Agarwal S, Upadhyay GC. Molecular epidemiology and therapeutic options of carbapenem-resistant Gram-negative bacteria. Indian J Med Res 2019; 149: 285-289.

22. Al-Ouqaili MTS, Jal'oot AS, Badawy AS. Identification of an OprD and blaIMP Gene-mediated carbapenem resistance in Acinetobacter baumannii and Pseudomonas aeruginosa among patients with wound infections in Iraq. Asian Journal of Pharmaceutics 2018; 12: S959.
23. Naim H, Rizvi M, Gupta R, Azam M, Taneja N, Shukla I, et al. Drug resistance and molecular epidemiology of carbapenem resistant Gram-negative bacilli isolates. J Glob Infect Dis 2018; 10: 133-139.

24. Otter JA, Burgess P, Davies F, Mookerjee S, Singleton J, Gilchrist $\mathrm{M}$, et al. Counting the cost of an outbreak of carbapenemaseproducing Enterobacteriaceae: an economic evaluation from a hospital perspective. Clin Microbiol Infect 2017; 23: 188-196.

25. Mohanam L, Menon T. Coexistence of metallo-beta-lactamaseencoding genes in Pseudomonas aeruginosa. Indian J Med Res 2017; 146: S46-S52.

26. Ellappan K, Belgode Narasimha H, Kumar S. Coexistence of multidrug resistance mechanisms and virulence genes in carbapenem-resistant Pseudomonas aeruginosa strains from a tertiary care hospital in South India. J Glob Antimicrob Resist 2018; 12: 37-43.

27. Vatansever C, Menekse S, Dogan O, Gucer LS, Ozer B, Ergonul $\mathrm{O}$, et al. Co-existence of OXA-48 and NDM-1 in colistin resistant Pseudomonas aeruginosa ST235. Emerg Microbes Infect 2020; 9: 152-154.

28. Esther J, Edwin D, Uma. Prevalence of carbapenem resistant non-fermenting Gram-negative bacterial infection and identification of carbapenemase producing NFGNB isolates by simple phenotypic tests. J Clin Diagn Res 2017; 11: DC10-DC13.

29. Pan F, Tian D, Wang B, Zhao W, Qin H, Zhang T, et al. Fecal carriage and molecular epidemiology of carbapenem-resistant Enterobacteriaceae from outpatient children in Shanghai. BMC Infect Dis 2019; 19: 678. 